Artigo Original

Original Article

Giseli Donadon Germano ${ }^{1}$

Fábio Henrique Pinheiro²

Paola Matiko Martins Okuda ${ }^{3}$

Simone Aparecida Capellini ${ }^{1,2}$

Descritores

Transtorno do déficit de atenção com hiperatividade Percepção visual Destreza motora Avaliação Educação

Keywords

Attention deficit disorder with hyperactivity Visual perception

Motor skills Evaluation Education

Endereço para correspondência: Giseli Donadon Germano Departamento de Fonoaudiologia Av. Hygino Muzzy Filho, 737, Campus Universitário, Marília (SP), Brasil, CEP: 17525-900.

E-mail: giseliger@yahoo.com.br

Recebido em: 05/12/2011

Aceito em: 05/08/2013

\section{Percepção viso-motora de escolares com Transtorno do Déficit de Atenção com Hiperatividade}

\author{
Visual-motor perception in students with Attention \\ Deficit with Hyperactivity Disorder
}

\section{RESUMO}

Objetivo: Caracterizar e comparar as habilidades de percepção viso-motoras de escolares com Transtorno do Déficit de Atenção com Hiperatividade (TDAH) com escolares com bom desempenho acadêmico. Métodos: Participaram deste estudo 40 escolares na faixa etária de 7 anos a 10 anos e 8 meses, do $2^{\circ}$ ao $5^{\circ}$ ano do Ensino Fundamental de escolas públicas, divididos em GI (20 escolares com diagnóstico interdisciplinar de TDAH) do gênero masculino (100\%) e GII (20 escolares com bom desempenho escolar), pareados com o GI em idade, escolaridade e gênero. Os escolares foram submetidos ao Teste Evolutivo de Percepção Visual (DTVP-2). Resultados: Os escolares de GI apresentaram desempenho inferior na função de posição no espaço e closura visual (motricidade reduzida) e equivalente de idade inferior para percepção de motricidade reduzida em relação ao GII. Conclusão: As dificuldades em percepção viso-motora apresentadas pelos escolares de GI podem ser atribuídas não a um déficit primário, mas a um fenômeno secundário à desatenção que interfere de forma direta em seu desempenho de percepção viso-motora.

\begin{abstract}
Purpose: The aim of this study was to characterize and to compare the visual-motor perception of students with Attention Deficit with Hyperactivity Disorder (ADHD) with students with good academic performance. Methods: Forty students from $2^{\text {nd }}$ to $5^{\text {th }}$ grades of an elementary public school, male gender $(100 \%)$, aged between 7 and 10 years and 8 months old participated, divided into: GI (20 students with ADHD) and GII (20 students with good academic performance), paired according to age, schooling and gender with GI. The students were submitted to Developmental Test of Visual Perception (DTVP-2). Results: The students of GI presented low performance in spatial position and visual closure (reduced motor) and inferior age equivalent in reduced motor perception, when compared to GII. Conclusion: The difficulties in visual-motor perception presented by students of GI cannot be attributed to a primary deficit, but to a secondary phenomenon of inattention that interferes directly in their visual-motor performance.
\end{abstract}

Trabalho realizado no Laboratório de Investigação dos Desvios da Aprendizagem, Centro de Estudos da Educação e Saúde, Faculdade de Filosofia e Ciências, Universidade Estadual Paulista "Júlio de Mesquita Filho" UNESP - e no Ambulatório de Neurologia Infantil-Desvios da Aprendizagem, Hospital das Clínicas, Faculdade de Medicina, Universidade Estadual Paulista "Júlio de Mesquita Filho" - UNESP - Botucatu (SP), Brasil.

(1) Departamento de Fonoaudiologia, Faculdade de Filosofia e Ciências, Universidade Estadual Paulista "Júlio de Mesquita Filho" - UNESP - Marília (SP), Brasil.

(2) Programa de Pós-Graduação em Educação, Faculdade de Filosofia e Ciências, Universidade Estadual Paulista "Júlio de Mesquita Filho" - UNESP - Marília (SP), Brasil.

(3) Laboratório de Investigação dos Desvios da Aprendizagem, Departamento de Fonoaudiologia, Faculdade de Filosofia e Ciências, Universidade Estadual Paulista "Júlio de Mesquita Filho" - UNESP - Marília (SP), Brasil. Conflito de interesses: nada a declarar. 


\section{INTRODUÇÃO}

A aquisição da escrita manual exige uma combinação de coordenação de habilidades viso-motoras com o planejamento motor, cognitivo e habilidades perceptuais (tátil-cinestésicas, organização no espaço e no tempo). A integração viso-motora é definida como a habilidade em coordenar informações visuais com a programação motora, sendo uma importante variável no desempenho da escrita. Por meio dela, o escolar consegue realizar cópia ou transposição de textos, letra cursiva, reprodução de letras e números isolados e em sequências ${ }^{(1,2)}$.

A percepção visual requer a conjugação da atenção voluntária e da capacidade de programação e reprogramação dos órgãos que irão realizar a atividade motora. Assim, a eficácia da velocidade de programação ocorre à medida em que as informações tátil-perceptivas se ajustam às informações visuais ${ }^{(3-5)}$, devido à integridade de estruturas corticais ${ }^{(6-9)}$.

O escolar que não desenvolve essa habilidade integrativa viso-motora poderá apresentar dificuldades para escrever, ou seja, dificuldades quanto à qualidade da escrita, prejudicando o progresso escolar e favorecendo o aparecimento de problemas emocionais, comportamentais e de aprendizagem ${ }^{(3)}$. Na literatura internacional $^{(10,11)}$ e na literatura nacional ${ }^{(12,13)}$, há descrição da relação entre a dislexia, transtorno de aprendizagem e alterações de coordenação motora fina e grossa como também a relação entre as alterações de percepção viso-motoras e o desempenho da leitura de escolares com esses transtornos de aprendizagem.

A literatura ${ }^{(14,15)}$ também vem descrevendo ao longo dos anos que uma das condições neurológicas que compromete a integração viso-motora é o Transtorno do Déficit de Atenção e Hiperatividade (TDAH). O TDAH é o distúrbio neuropsiquiátrico mais comum da infância, que afeta entre 3 e $6 \%$ das crianças em idade escolar, apresentando como principais manifestações a dificuldade em atenção, a hiperatividade e/ou a impulsividade, afetando frequentemente diversas áreas do funcionamento adaptativo, nomeadamente interpessoal, acadêmico ou familiar ${ }^{16,17)}$.

Dentre as manifestações clínicas fonoaudiológicas apresentadas pelos escolares com TDAH, encontra-se o déficit da percepção viso-espacial, relacionado com disfunções executivas, agitação psicomotora e qualidade da escrita alterada, conhecida como escrita ininteligível ou disgrafia. Assim, devido a essas manifestações, o escolar com TDAH apresenta déficits de integração da percepção viso-motora que podem ser devido às alterações atencionais e às dificuldades da percepção viso-espacial, da função executiva, da organização perceptiva, do sincronismo, do atraso da maturação da coordenação e da dispraxia construtiva ${ }^{(18-20)}$.

Além dessas alterações, os escolares com TDAH tendem a ter dificuldades em tarefas de coordenação motora fina (pegar objetos, abotoar roupas, jogar bola, colorir dentro dos limites de figuras, escrever sobre a linha em tamanho uniforme, escrever com letra compreensível) e global (dificuldades para correr ou saltar e problemas com a lateralidade $)^{(19)}$ e essas dificuldades podem estar relacionadas com alterações percepto-viso-motoras ${ }^{(18,20)}$, facilmente identificáveis durante a avaliação fonoaudiológica e no contexto educacional.

Dentre as manifestações fonoaudiológicas mais perceptíveis no TDAH, destaca-se a disgrafia, que é definida como um transtorno da expressão escrita, que resulta em habilidades de escrita abaixo do esperado para a idade, relacionada à legibilidade (qualidade da formação da letra, alinhamento e espaçamento de letras e palavras, dimensionamento das letras) e à velocidade reduzida (taxa de produção) $)^{(3,4,10,13,21)}$.

Apesar de as razões para a ocorrência da disgrafia estarem associadas com planejamento motor, coordenação olho-mão, percepção visual, integração viso-motora, percepção cinestésica, controle motor fino, atenção sustentada e manipulação com as mãos ${ }^{(4,10,20)}$, ainda se tornam necessários estudos mais aprofundados acerca do papel das habilidades visuais e das habilidades perceto-viso-motoras para a determinação de um traçado de letra ininteligível, ou seja, o quadro de disgrafia, pois ainda são restritos esses estudos na literatura nacional, seja com escolares em fase final de escolarização ou com escolares com problemas de atenção e aprendizagem.

Com base no exposto, este estudo teve como objetivo caracterizar e comparar as habilidades de percepção viso-motoras de escolares com o diagnóstico de TDAH e escolares com bom desempenho acadêmico.

\section{MÉTODOS}

Este estudo foi aprovado pelo Comitê de Ética em Pesquisa da instituição de origem, sob o protocolo de número 0149/2011.

Participaram deste estudo 40 escolares na faixa etária de 7 anos a 10 anos e 8 meses, com nível socioeconômico médio, com base no estudo estatístico do Índice de Desenvolvimento Socioeconômico ${ }^{(22)}$ do Ensino Fundamental de escolas públicas municipais. Os escolares foram divididos em dois grupos:

- Grupo I (GI): 20 escolares com diagnóstico interdisciplinar de TDAH, seguindo critérios propostos ${ }^{(23)}$, do gênero masculino (100\%), com uso de medicação (metilfenidato) indicada pelo neurologista, pelo menos, por seis meses. Os escolares deste grupo apresentavam desempenho acadêmico insatisfatório, aferido com nota igual ou inferior a cinco em avaliações de português (avaliação de leitura, de escrita e cópia) e de matemática (operações aritméticas com e sem enunciado). As avaliações foram realizadas pelos professores em sala de aula, os quais referiram escrita ininteligível. Os escolares não apresentavam histórico de terapias fonoaudiológicas ou pedagógicas anteriores a este estudo e faziam parte da lista de espera para intervenção fonoaudiológica de leitura e escrita da instituição de origem.

- Grupo II (GII): 20 escolares com bom desempenho escolar em dois bimestres seguidos, com desempenho acadêmico satisfatório, aferido com nota superior a cinco em avaliações de português (avaliação de leitura, de escrita e cópia) e de matemática (operações aritméticas com e sem enunciado), realizadas pelos professores em ambiente de sala de aula. Os escolares do GII que participaram desta pesquisa foram indicados pelos professores e pareados com o GI segundo a idade, a escolaridade e o gênero. Participaram deste estudo somente escolares que apresentaram a assinatura do termo de consentimento e que não apresentaram intercorrências pré, peri e pós-natais ou atraso no desenvolvimento neuropsicomotor e linguagem, descritos em prontuário escolar.

Todos os escolares foram submetidos ao Teste Evolutivo de Percepção Visual, segunda edição (DTVP-2) ${ }^{(24)}$, composto de oito subtestes que medem habilidades viso-motoras 
interrelacionadas com as habilidades de percepção visuais diferentes. Sua fidedignidade e validade foram empiricamente estabelecias ${ }^{(23)}$ e o procedimento não tem níveis basais, uma vez que a testagem inicia-se com o item 1 de cada subteste.

O DTVP-2 consiste em uma bateria de oito subtestes que medem habilidades viso-motoras e percepto-visuais diferentes, porém inter-relacionadas. Desse modo, cada um dos oito subtestes mede um tipo de capacidade percepto-visual, quais sejam a posição no espaço, a constância da forma, as relações espaciais ou a figura-fundo. Por outro lado, cada subteste pode ser classificado como de motricidade reduzida ou de motricidade plena ${ }^{(24)}$.

Os subtestes que compõem o DTVP-2 são: coordenação visomotora (CVM), posição no espaço (PE), cópia $(\mathrm{C})$, figura-fundo (FF), relações espaciais (RE), closura visual (CV), velocidade viso-motora (VVM) e constância de forma (CF). Todos os subtestes medem um tipo de capacidade de percepção visual e podem ser considerados como subteste de motricidade reduzida (posição no espaço, figura-fundo, closura visual e constância de forma) e de motricidade plena ou integração viso-motora (coordenação viso-motora, cópia, relações espaciais, velocidade viso-motora).

Sua pontuação é dividida em: escore padrão, que é obtido a partir da pontuação bruta e sua conversão mediante utilização de tabelas, e escore composto, obtido por meio da soma dos escores padrão e sua conversão em um quociente classificatório em relação à percepção visual geral, percepção de motricidade reduzida e integração viso-motora. Todas as funções avaliadas permitem o cálculo de um equivalente de idade (EI), ou seja, para cada função avaliada, o escore obtido permite o cálculo de uma "idade de percepção visual".

Os resultados obtidos foram analisados estatisticamente por meio do Teste de Mann-Whitney, para verificar possíveis diferenças de desempenho nas tarefas entre os grupos estudados, e do Teste da Razão de Verossimilhança, com o objetivo de comparar os desempenhos entre os subtestes de ambos os grupos deste estudo. O nível de significância (valor de p) adotado foi de 5\% $(0,050)$ e está marcado com asterisco. Para a análise dos dados, foi utilizado o programa Statistical Package for the Social Sciences (SPSS), em sua versão 17.0.

\section{RESULTADOS}

A Tabela 1 apresenta a média, desvio-padrão, mediana e valor de p referentes à comparação entre os grupos GI e GII e os resultados dos subtestes de percepção viso-motora, analisados pelo Teste de Mann-Whitney.

Na Tabela 1, foi possível observar que houve diferença entre os grupos GI e GII, quando comparados em seus desempenhos por subteste, demonstrando que GI apresentou desempenho inferior na função de posição no espaço (PE) e closura visual (CV) e GII, na função de velocidade viso-motora (VVM).

A Tabela 2 apresenta a média, desvio-padrão e significância da comparação entre os grupos GI e GII nos subtestes de percepção visual geral, percepção de motricidade reduzida, integração viso-motora e em relação à média dos equivalentes de idade em cada uma das funções, para cada grupo, analisados pelo Teste de Mann-Whitney.

Na Tabela 2, foi possível notar que houve diferença entre os grupos GI e GII, quando comparados em seus desempenhos gerais,
Tabela 1. Comparação de desempenho entre os grupos Gl e Gll nos subtestes de percepção viso-motora

\begin{tabular}{|c|c|c|c|c|c|c|}
\hline Variável & Grupo & $\mathrm{n}$ & Média & $\begin{array}{l}\text { Desvio- } \\
\text { padrão }\end{array}$ & Mediana & Valor de $\mathrm{p}^{*}$ \\
\hline & I & 20 & 145,60 & 18,63 & 146,50 & \multirow{3}{*}{0,725} \\
\hline \multirow[t]{2}{*}{ CVM } & II & 20 & 145,35 & 28,19 & 150,00 & \\
\hline & Total & 40 & 145,48 & 23,58 & 147,50 & \\
\hline \multirow{3}{*}{ PE } & I & 20 & 14,05 & 6,67 & 15,00 & \multirow{3}{*}{$0,015^{\star}$} \\
\hline & II & 20 & 18,45 & 6,85 & 20,50 & \\
\hline & Total & 40 & 16,25 & 7,03 & 17,50 & \\
\hline \multirow{3}{*}{ C } & $\mathrm{I}$ & 20 & 26,80 & 5,51 & 26,50 & \multirow{3}{*}{0,171} \\
\hline & II & 20 & 28,65 & 5,53 & 30,50 & \\
\hline & Total & 40 & 27,73 & 5,53 & 28,50 & \\
\hline \multirow{3}{*}{ FF } & I & 20 & 8,95 & 2,74 & 8,50 & \multirow{3}{*}{0,343} \\
\hline & II & 20 & 9,75 & 2,88 & 9,00 & \\
\hline & Total & 40 & 9,35 & 2,81 & 9,00 & \\
\hline \multirow{3}{*}{ RE } & $\mathrm{I}$ & 20 & 32,00 & 11,80 & 36,00 & \multirow{3}{*}{0,289} \\
\hline & II & 20 & 38,75 & 6,30 & 42,00 & \\
\hline & Total & 40 & 35,38 & 9,94 & 40,50 & \\
\hline \multirow{3}{*}{ CV } & 1 & 20 & 6,95 & 4,27 & 6,00 & \multirow{3}{*}{$0,011^{*}$} \\
\hline & II & 20 & 10,85 & 5,35 & 11,50 & \\
\hline & Total & 40 & 8,90 & 5,17 & 8,00 & \\
\hline \multirow{3}{*}{ VVM } & I & 20 & 14,40 & 9,27 & 13,00 & \multirow{3}{*}{$0,009^{*}$} \\
\hline & II & 20 & 7,35 & 3,84 & 8,00 & \\
\hline & Total & 40 & 10,88 & 7,86 & 9,00 & \\
\hline \multirow{3}{*}{ CF } & $\mathrm{I}$ & 20 & 8,95 & 3,98 & 9,00 & \multirow{3}{*}{0,348} \\
\hline & II & 20 & 10,10 & 3,37 & 9,50 & \\
\hline & Total & 40 & 9,53 & 3,69 & 9,00 & \\
\hline
\end{tabular}

*Valores significativos - Teste de Mann-Whitney

Legenda: $C V M=$ coordenação viso-motora; $P E$ = posição no espaço; $C$ = cópia; $\mathrm{FF}=$ figura-fundo; $\mathrm{RE}=$ relação espacial; $\mathrm{CV}=$ closura visual; $\mathrm{VVM}=$ velocidade viso-motora; $\mathrm{CF}=$ constância de forma

Tabela 2. Comparação de desempenho entre os grupos GI e Gll nos subtestes de percepção visual geral, percepção de motricidade reduzida e integração viso-motora em relação aos equivalentes de idade em cada uma das funções

\begin{tabular}{|c|c|c|c|c|c|}
\hline Variável & Grupo & $\mathrm{n}$ & Média & Desvio-padrão & Valor de $\mathrm{p}^{*}$ \\
\hline & $\mathrm{I}$ & 20 & 8,88 & 1,06 & \\
\hline \multirow[t]{3}{*}{ IC } & II & 20 & 8,85 & 1,01 & 0,850 \\
\hline & Total & 40 & 8,86 & 1,02 & \\
\hline & 1 & 20 & 80,70 & 15,67 & \\
\hline \multirow[t]{2}{*}{ PVG } & II & 20 & 86,75 & 8,93 & 0,068 \\
\hline & Total & 40 & 83,73 & 12,96 & \\
\hline \multirow{3}{*}{ EI PVG } & $\mathrm{I}$ & 20 & 6,65 & 1,72 & \\
\hline & II & 20 & 7,39 & 1,77 & 0,148 \\
\hline & Total & 40 & 7,02 & 1,76 & \\
\hline & I & 20 & 72,40 & 16,30 & \\
\hline \multirow[t]{3}{*}{ PMR } & II & 20 & 84,50 & 12,80 & $0,005^{\star}$ \\
\hline & Total & 40 & 78,45 & 15,71 & \\
\hline & $\mathrm{T}$ & 20 & 5,41 & 1,69 & \\
\hline \multirow[t]{2}{*}{ El PMR } & II & 20 & 6,96 & 1,95 & $0,004^{*}$ \\
\hline & Total & 40 & 6,18 & 1,97 & \\
\hline \multirow{3}{*}{ IMV } & $\mathrm{T}$ & 20 & 87,60 & 18,73 & \\
\hline & II & 20 & 89,95 & 10,35 & 0,735 \\
\hline & Total & 40 & 88,78 & 14,98 & \\
\hline \multirow{3}{*}{ EI IMV } & $\mathrm{T}$ & 20 & 7,94 & 2,02 & \\
\hline & II & 20 & 7,85 & 1,63 & 0,903 \\
\hline & Total & 40 & 7,89 & 1,81 & \\
\hline
\end{tabular}

*Valores significativos - Teste de Mann-Whitney

Legenda: $\mathrm{IC}$ = idade cronológica; $P V G$ = percepção visual geral; $E \mathrm{EI}$ = equivalente de idade; PMR = percepção de motricidade reduzida; $I M V$ = integração viso-motora

demonstrando que GI apresentou desempenho inferior nas provas de percepção de motricidade reduzida (PMR) e equivalente de idade inferior para percepção de motricidade reduzida em relação ao GII.

A Tabela 3 mostra a comparação da classificação do desempenho em percepção visual geral, percepção de motricidade reduzida e integração viso-motora entre os grupos I e II. 
Tabela 3. Comparação entre os grupos GI e Gll da classificação do desempenho em percepção visual geral, percepção de motricidade reduzida e integração viso-motora

\begin{tabular}{|c|c|c|c|c|c|c|c|c|}
\hline & \multirow{2}{*}{ Grupo } & \multicolumn{5}{|c|}{ Classificação do desempenho } & \multirow{2}{*}{ Total } & \multirow{2}{*}{ Valor de $p$} \\
\hline & & 1 & 2 & 3 & 4 & 6 & & \\
\hline & $\mathrm{I}$ & $5(25 \%)$ & $5(25 \%)$ & $5(25 \%)$ & $4(20 \%)$ & $1(5 \%)$ & $20(100 \%)$ & \\
\hline \multirow[t]{3}{*}{ PVG } & II & $2(10 \%)$ & $2(10 \%)$ & $8(40 \%)$ & $8(40 \%)$ & $0(0 \%)$ & $20(100 \%)$ & 0,192 \\
\hline & Total & $7(17,5 \%)$ & $7(17,5 \%)$ & $12(30 \%)$ & $1(2,5 \%)$ & $1(2,5 \%)$ & $40(100 \%)$ & \\
\hline & 1 & $8(40 \%)$ & $8(40 \%)$ & $3(15 \%)$ & $0(0 \%)$ & $1(5 \%)$ & $20(100 \%)$ & \\
\hline \multirow[t]{3}{*}{ PMR } & II & $4(20 \%)$ & $3(15 \%)$ & $4(20 \%)$ & $9(45 \%)$ & $0(0 \%)$ & $20(100 \%)$ & $0,001^{*}$ \\
\hline & Total & $12(30 \%)$ & $11(27,5 \%)$ & $7(17,5 \%)$ & $9(22,5 \%)$ & $1(2,5 \%)$ & $40(100 \%)$ & \\
\hline & 1 & $5(25 \%)$ & $1(5 \%)$ & $6(30 \%)$ & $6(30 \%)$ & $2(10 \%)$ & $20(100 \%)$ & \\
\hline \multirow[t]{2}{*}{ IMV } & II & $1(5 \%)$ & $2(10 \%)$ & 5 (25\%) & $12(60 \%)$ & $0(0 \%)$ & $20(100 \%)$ & 0,086 \\
\hline & Total & $6(15 \%)$ & $3(7,5 \%)$ & $11(27,5 \%)$ & $18(45 \%)$ & $2(5 \%)$ & $40(100 \%)$ & \\
\hline
\end{tabular}

*Valores significativos - Teste da Razão de Verossimilhança

Legenda: $P V G$ = percepção visual geral; PMR = percepção de motricidade reduzida; IMV = integração viso-motora; 1 = muito fraco; 2 = fraco; 3 = abaixo da média; 4 = médio; 5 = acima da média; 6 = muito bom

Na Tabela 3, observou-se que os resultados das classificações das percepções visuais, analisados pelo Teste da Razão de Verossimilhança, demonstraram que houve diferença entre o GI e GII somente em percepção de motricidade reduzida, demonstrando que a classificação obtida pelos escolares do GI é inferior à classificação obtida pelos escolares do GII.

A classificação dos desempenhos foi convertida em formas numéricas $(1=$ muito fraco; 2 = fraco; $3=$ abaixo da média; 4 = médio; 5 = acima da média; $6=$ muito bom), a fim de facilitar a análise estatística dos dados. Assim, ao observarmos a Tabela 3, não encontramos análises relacionadas à classificação de desempenho em percepção visual 5 (acima da média), em percepção visual geral (PVG) e em percepção de motricidade reduzida (PMR), bem como não encontramos análises relacionadas à classificação de desempenho em percepção visual 6 (muito bom) em integração viso-motora (IVM), pois nenhum dos escolares obtiveram essas classificações.

\section{DISCUSSÃO}

Com base nos dados obtidos, pôde-se observar que os escolares com TDAH apresentaram desempenho inferior em relação aos escolares com bom desempenho acadêmico, referindo-se às funções de posição no espaço e closura visual, relacionadas à motricidade reduzida (percepção visual sem componentes motores), obtendo a classificação de desempenho muito fraco e fraco predominantemente e tendo desempenhos inferiores nas habilidades de posição no espaço e closura visual, em relação aos escolares com bom desempenho acadêmico.

Feder e Majnemer ${ }^{(3)}$ referiram que a habilidade de posição no espaço permite que o escolar perceba e identifique espaços entre letras, entre palavras e entre as linhas, enquanto que a habilidade de closura visual permite que o escolar identifique quais letras foram escritas completamente.

Sendo assim, pode-se afirmar que os escolares com TDAH deste estudo apresentam alterações de percepção viso-motoras, corroborando estudos descritos na literatura internacional $^{(2,14)}$ e nacional ${ }^{(13,25)}$. Assim, podemos considerar que as dificuldades na execução das habilidades de percepção viso-motora e percepção visual (motricidade reduzida) presentes nesses escolares podem comprometer o desempenho em escrita manual, ocasionando a disgrafia, conforme descrito na literatura ${ }^{(21,26)}$.

As habilidades de percepção viso-motoras estão diretamente relacionadas com a escrita manual, ou seja, na ação grafo-motora e também na habilidade de leitura, pois dependem do reconhecimento de detalhes, de processamento simultâneo, da organização viso-espacial, da relação espacial entre figuras e de integração das partes de um todo, dando significados às formas das letras e, consequentemente, afetando o desempenho motor (grafo-motor) para a produção dessas letras, causando dificuldades nas aquisições de habilidades escolares básicas de leitura e escrita ${ }^{(20,26-29)}$.

Brown, Unsworth e Lyons ${ }^{(2)}$ e Feder e Majnemer ${ }^{(3)}$ referiram que o baixo desempenho nas habilidades anteriormente citadas acarreta dificuldades em coordenação motora fina e global, leitura, matemática, problemas perceptuais e em outras áreas acadêmicas.

Assim, para os escolares com TDAH deste estudo, o desempenho inferior na habilidade motora fina corrobora a literatura, a qual referiu que tal desempenho pode ser atribuído ao déficit da velocidade do movimento e à imaturidade do desenvolvimento da coordenação, ambos subordinados à coordenação cerebral de grupos alternados de músculos ${ }^{(30)}$, indicando, assim, que os resultados encontrados neste estudo podem permitir ao fonoaudiólogo uma maior reflexão acerca das relações entre as habilidades de percepção viso-motora e a qualidade de escrita disgráfica de escolares com TDAH.

No entanto, também devemos considerar que, além das habilidades viso-motoras analisadas neste artigo, outros fatores como a impulsividade ou controle de inibição e a auto-regulação, relacionados às funções executivas, também são fatores importantes a serem analisados e considerados, pois são responsáveis pelo planejamento motor; desta forma, a investigação desses aspectos também deve ser realizada no momento da avaliação clínica fonoaudiológica, principalmente se uma das manifestações do escolar com TDAH for a escrita ininteligível. 


\section{CONCLUSÃO}

Os escolares com TDAH deste estudo apresentaram desempenho inferior em habilidades de percepção viso-motora, quando comparados aos escolares com bom desempenho acadêmico, caracterizados pelas alterações em percepção de motricidade reduzida (posição no espaço, figura-fundo, closura visual e constância de forma) e alterações em integração viso-motora (coordenação viso-motora, cópia, relação espacial e velocidade viso-motora).

*GDG foi responsável pela elaboração da proposta de pesquisa, análise dos resultados e redação do manuscrito; FHP realizou a coleta de dados; PMMO realizou a coleta, tabulação e análise de dados; SAC foi responsável pelo projeto e delineamento do estudo e orientação geral das etapas de execução e elaboração do manuscrito.

\section{REFERÊNCIAS}

1. Brown T, Rodger S. Validity of the Developmental Test of Visual-Motor Integration Supplemental Developmental Test of Visual Perception. Percept Mot Skills. 2008;106(3):659-78.

2. Brown T, Unsworth C, Lyons C. Factor structure of four visual-motor instruments commonly used to evaluate school-age children. Am J Occup Ther. 2009;63(6):710-23.

3. Feder KP, Majnemer A. Handwriting development, competency, and intervention. Dev Med Child Neurol. 2007;49(4):312-7.

4. Rosenblum S, Aloni T, Josman N. Relationships between handwriting performance and organizational abilities among children with and without dysgraphia: a preliminary study. Res Dev Disabil. 2010;31(2):502-9.

5. Miller AD, Goodale MA. Two visual systems re-viewed. Neuropsychologia. 2008;46(3):774-85.

6. Huberle E, Driver J, Karnath HO. Retinal versus physical stimulus size as determinants of visual perception in simultanagnosia. Neuropsychologia. 2010;48(6):1677-82.

7. Qiu J, Li H, Liu Q, Zhang Q. Brain mechanisms underlying visual perception and visual mental imagery of Chinese pseudo-characters: an event-related potential study. Brain Res. 2007;1184:202-9.

8. Khayat PS, Pooresmaeili A, Roelfsema PR. Time Course of Attentional Modulation in the Frontal Eye Field During Curve Tracing. J Neurophysiol. 2009;101(4):1813-22.

9. Wardak $\mathrm{C}$, Denève $\mathrm{S}$, Ben Hamed $\mathrm{S}$. Focused visual attention distorts distance perception away from the attentional locus. Neuropsychologia. 2011;49(3):535-45.

10. Rosemblum S. Development, reliability, and validity of the Handwriting Proficiency Screening Questionnaire (HPSQ). Am J Occup Ther. 2008;62(3):298-307.

11. Loh PR, Piek JP, Barrett NC. Comorbid ADHD and DCD: examining cognitive functions using the WISC-IV. Res Dev Disabil. 2011;32(4):1260-9.

12. Siqueira CM, Gurgel-Giannetti J. Poor school performance: an updated review. Rev Assoc Med Bras. 2011;57(1):78-87.

13. Okuda PMM, Lourencetti MD, Santos LCA, Padula NAMR, Capellini SA. Coordenação motora fina de escolares com dislexia e transtorno do déficit de atenção e hiperatividade. Rev. CEFAC. 2011;13(5):876-85.
14. Crawford SG, Dewey D. Co-occurring disorders: a possible key to visual perceptual deficits in children with developmental coordination disorder? Hum Mov Sci. 2008;27(1):154-69.

15. Henríquez-Henríquez M, Zamorano-Mendieta F, Rothhammer-Engel F, Aboitiz F. Modelos neurocognitivos para el trastorno por déficit de atención/hiperactividad y sus implicaciones en el reconocimiento de endofenotipos. Rev Neurol. 2010;50(2):109-16.

16. Fliers E, Rommelse N, Vermeulen SHHM, Altink M, Buschgens CJM, Faraone SV, et al. Motor coordination problems in children and adolescents with ADHD rated by parents and teachers: effects of age and gender. J Neural Transm. 2008;115:211-20.

17. Oliveira CG, Albuquerque PB. Diversidade de resultados no estudo do Transtorno de Déficit de Atenção e Hiperatividade. Psicol. Teor Pesqui. 2009;25(1):93-102.

18. Racine MB, Shevell M, Snider L. Handwriting in children with Attention Deficit Hyperactivity Disorder (ADHD). J Child Neurol. 2008;23(4):399-406.

19. Vidarte JA, Ezquerro M, Giráldez MA. Perfil psicomotor de niños de 5 a 12 años diagnosticados clínicamente de trastorno por déficit de atención/ hiperactividad en Colombia. Rev neurol. 2009;49(2):69-75.

20. Ratzon NZ, Lahav O, Cohen-Hamsi S, Metzger Y, Efraim D, Bart O. Comparing different short-term service delivery methods of visual-motor treatment for first grade students in mainstream schools. Res Dev Disabil. 2009;30(6):1168-76.

21. Toniolo CS, Santos LCA, Lourenceti MD, Padula NAMR, Capellini SA. Caracterização do desempenho motor em escolares com Transtorno do Déficit de Atenção com Hiperatividade. Rev Psicopedagogia. 2009;26(79):33-40.

22. FEE: Fundação de Economia e Estatística [Internet]. Índice de Desenvolvimento Socioeconômico (IDESE) [Acesso em: 2013 Ago 06]. Disponível em: http://www.fee.tche.br/sitefee/pt/content/estatisticas/ pg_idese.php

23. APA: Associação Americana de Psiquiatria. Manual diagnóstico estatístico de transtornos mentais (DSM-IV-TR). Porto Alegre: Artes Médicas; 2002.

24. Hammil DD, Pearson NA, Voress JK. Teste evolutivo de percepção visual. $2^{\mathrm{a}}$ ed. Ferreira MC, tradutora. Rio de Janeiro: Entreletras; 2001.

25. Pinheiro FH, Lourenceti MD, Santos LCA. Transtorno do déficit de atenção e hiperatividade: critérios diagnósticos. In: Capellini AS, Germano GD, Cunha VLO. Transtornos de aprendizagem e transtornos da atenção (da avaliação à intervenção). São José dos Campos: Pulso Editorial; 2010. p. 91-103.

26. Racine MB, Majnemer A, Shevell M, Snider L. Handwriting performance in children with Attention Deficit Hyperactivity Disorder (ADHD). J Child Neurol. 2008;23(4):399-406.

27. James KH, Gauthier I. When writing impairs reading: letter perception's susceptibility to motor interference. J Exp Psychol Gen. 2009;138(3):416-31.

28. Kushki A, Schwellnus H, Ilyas F, Chau T. Changes in kinetics and kinematics of handwriting during a prolonged writing task in children with and without dysgraphia. Res Dev Disabil. 2011;32(3):1058-64.

29. Falk TH, Tam C, Schellnus H, Chau T. On the development of a computer-based handwriting assessment tool to objectively quantify handwriting proficiency in children. Comput Methods Programs Biomed. 2011;104(3):e102-11.

30. Stray LL, Stray T, Iversen S, Ruud A, Ellertsen B, Tønnessen FE. The Motor Function Neurological Assessment (MFNU) as an indicator of motor function problems in boys with ADHD. Behav Brain Funct. 2009;5:22. 